\title{
Construcción sismorresistente en tierra: la gran experiencia contemporánea de la Pontificia Universidad Católica del Perú
}

\author{
Seismic resistant earthen Construction: the contemporary \\ experience at the Pontificia Universidad Católica del Perú
}

M. Blondet $^{(*)}$, J. $\operatorname{Vargas}^{(*)}$, N. Tarque ${ }^{(* *)}$, C. Iwaki $i^{(*)}$

\section{RESUMEN}

En muchos países en vías de desarrollo la alternativa de vivienda más común es la construcción con tierra, pues el material es abundante y barato. La construcción tradicional de viviendas de tierra se realiza informalmente, sin asesoría técnica. Por ello, la calidad de estas construcciones es generalmente muy baja. En zonas sísmicas donde se construye con tierra, cada vez que ocurre un terremoto colapsan muchas construcciones de este material, causando considerables pérdidas económicas y lamentables pérdidas de vidas. Las comunidades académicas y profesionales de algunos países sísmicos no han permanecido impasibles frente a esta grave situación. En el Perú, investigadores de la Pontificia Universidad Católica del Perú (PUCP) han venido investigando la construcción con tierra en áreas sísmicas desde hace cerca 40 años y han obtenido invalorables resultados.

Este artículo describe la evolución del conocimiento generado en la PUCP del material tierra armada, compuesto por tierra y refuerzos compatibles, que permite lograr construcciones sismorresistentes.

\section{SUMMARY}

In many developing countries the most common alternative for dwelling construction is building with earth because the material is abundant and cheap. The quality of most traditional earthen construction is very low, because it is done informally, with little or no technical assistance. In seismic areas where earthen construction is common, every time an earthquake occurs, many dwellings collapse, causing considerable economic losses and tragic injuries and deaths. The professional and academic communities in some seismic countries have not remained indifferent to this important problem. Researchers at the Catholic University of Peru $(P \cup C P)$ have been investigating on the construction of earthen buildings in seismic areas for about 40 years and have obtained invaluable results.

This article describes the evolution of the knowledge generated at PUCP of reinforced earth, a material composed of earth and compatible reinforcements, with which it is possible to build seismic-resistant constructions.
Palabras clave: adobe, tierra, sismo, refuerzo, tradicional, restauración, muros históricos.
Keywords: adobe, earth, seism, reinforce, restoration, historical walls. 

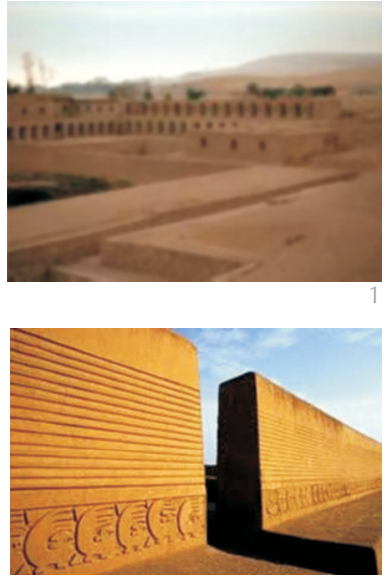

1. Pachacamac, Perú. Fuente: www. peruhotel.com

2. Muros decorados de Chan Chan, Perú. Fuente: http://www.inkas.com/ tours/jpg files/jpg photos/northern kingdoms/trujillo_chan_chan.jpg

3. Iglesia colonial ubicada en Lima (Santa Rosa de Lima)

4. Casas coloniales y republicanas en Lima

\section{INTRODUCCIÓN}

Desde hace cerca de 40 años los profesores del Departamento de Ingeniería de la PUCP complementaron sus labores docentes con tareas de investigación. Desde el principio, se ha tenido la preocupación de investigar la estabilidad de las construcciones de tierra en áreas sísmicas con la finalidad de encontrar refuerzos sísmicos adecuados y de uso masivo.

La metodología seguida en este trabajo contempla la presentación y descripción de las investigaciones más relevantes de años de trabajo en adobe de la academia de la PUCP; además del análisis de la vulnerabilidad sísmica de las casas de tierra seguida luego del terremoto del 15 de agosto del 2007 en Pisco, Perú.

El objetivo principal es demostrar que el uso de refuerzos mejora notablemente el desempeño sísmico de las estructuras de adobe, logrando salvaguardar la vida de sus ocupantes.

\section{CONSTRUCCIÓN CON ADOBE EN EL PERÚ}

El Perú está ubicado en la zona más sísmica de Sudamérica, donde a largo del tiempo confluyeron varias culturas con tradición de construcción con tierra: las pre-incas, la inca y la española-árabe. Las tecnologías imperantes fueron la mampostería de adobe, el tapial (mampostería de tierra apisonada), y la quincha (bastidores de troncos de madera, caña o fibras vegetales, rellenos con tierra).
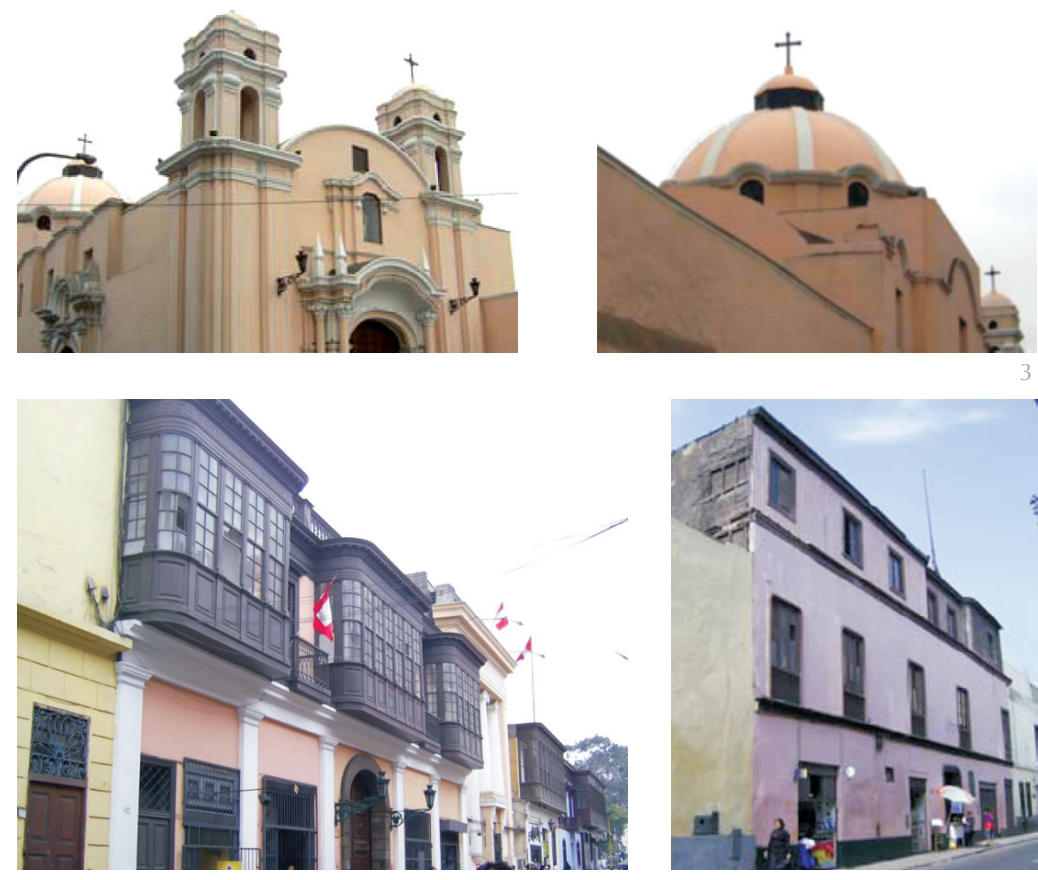

La ciudad de Caral, la más antigua de América (2800 a.C.) ubicada en la costa, 200 km al norte de Lima, muestra el empleo de estas tres tecnologías y de la durabilidad que alcanzaron sus obras.

Cerca de Lima, hacia el sur, se encuentran alrededor de 300 sitios de interés arqueológico, donde destaca el complejo arqueológico de Pachacamac, dios del fuego y de los terremotos. El templo del Acllawasi de Pachacamac, cuya construcción fue iniciada alrededor del siglo XIII, es una magnífica construcción Inca de adobe destruida por los sismos y reconstruida en 1945 (Figura 1). Hoy se encuentra nuevamente dañada por varios terremotos y constituye una muestra de la necesidad de encontrar un nuevo paradigma de conservación de obras de tierra en áreas sísmicas.

Otro ejemplo que merece mención es Chan Chan, una ciudadela construida íntegramente en tierra, ubicada en la ciudad de TrujiIlo, en la costa norte del Perú. Chan Chan surgió entre los años 600 y 700 DC, cubre aproximadamente un área de veinte kilómetros cuadrados, y contiene gran cantidad de ambientes, edificios, plazas, pirámides ceremoniales y depósitos. Se estima que sus palacios e instalaciones albergaron hasta alrededor de cien mil personas durante su apogeo en el siglo XV DC como capital del reino Chimú. Las obras de Chan Chan tienen un gran valor arquitectónico por sus formas y por sus muros decorados con altorrelieves (Figura 2).

La época colonial (1540 - 1821) se caracterizó también por el uso primordial y extensivo de la construcción con tierra. Durante la colonia, los españoles realizaron importantes obras de mampostería de adobe, tapial y posteriormente quincha. Muchas de las numerosas iglesias fueron construidas en tierra, con muros en adobe o tapial, y bóvedas y cúpulas en quincha (Figura 3).

Las casas coloniales de los españoles fueron también construidas en tierra y algunas tuvieron dos y hasta tres niveles. Después del gran sismo de 1746 una Real Ordenanza limitó las construcciones de adobe a un solo piso, pudiéndose construir pisos superiores en quincha. La quincha colonial, basada en la quincha autóctona, emplea bastidores de madera aserrada rellenos con caña y barro. Las casas de la Figura 4 tienen el primer piso de adobe y los pisos superiores de quincha.

Las casas coloniales de tierra y de la primera etapa republicana que han prevalecido a pesar de la alta sismicidad de la costa peruana son robustas pues tienen muros anchos en densidad suficiente en las dos direcciones 
principales. La construcción en tierra en el Perú ha continuado aumentando y hoy en día existen cerca de dos millones y medio de viviendas de tierra que representan cerca al $40 \%$ del inventario nacional (1). Lamentablemente, y debido principalmente a razones económicas, poco a poco se ha ido perdiendo la tradición de construir edificaciones de tierra y las nuevas viviendas de tierra son paulatinamente menos sismorresistentes, pues tienen muros más esbeltos e imitan configuraciones arquitectónicas de las viviendas de albañilería confinada.

\section{TIERRA SIMPLE}

La tierra como material constructivo tiene su origen en el descubrimiento que al humedecerse, la tierra que contiene arcilla es plástica y fácil de moldear. Luego, al secarse se endurece y puede alcanzar una resistencia importante a la compresión. La construcción con tierra se hace extensiva porque el material es fácilmente accesible y permite lograr espacios con bondades ambientales. Las casas de tierra tienen una alta capacidad de aislamiento acústico y térmico frente a situaciones extremas de frío o calor: son cálidas en invierno y frescas en verano. Su construcción sin consumo de energía agotable las convierte en edificaciones ecológicamente compatibles con la naturaleza. Las casas de tierra bien concebidas y construidas pueden ser bellas por sus formas rústicas y grandes espesores de muros y también, si son bien mantenidas, pueden ser perdurables como lo demuestran los numerosos ejemplos de construcciones históricas.

La tierra seca, gracias a la presencia de arcilla, ofrece una resistencia considerable a esfuerzos de compresión; sin embargo, tiene escasa resistencia a cargas que le produzcan esfuerzos de tracción. Los muros, que son los elementos estructurales más importantes de las construcciones de tierra, poseen buena capacidad resistente a cargas de gravedad. Cuando ocurre un sismo moderado o fuerte se generan en los muros (especialmente en los encuentros) esfuerzos de tracción que la tierra es incapaz de soportar. Los muros se agrietan y se separan en pedazos que luego se caen, arrastrando consigo a los techos. Las fallas sísmicas de los muros de tierra simple son frágiles, súbitas, y muchas veces fatales para sus habitantes (Figura 5).

La construcción de tierra típica en el Perú es de tierra simple, sin ningún refuerzo sísmico. Hoy se emplea principalmente en el área rural y en las zonas periurbanas de muchas ciudades. Existe también un importante stock de construcciones de tierra urbanas construidas en el pasado.

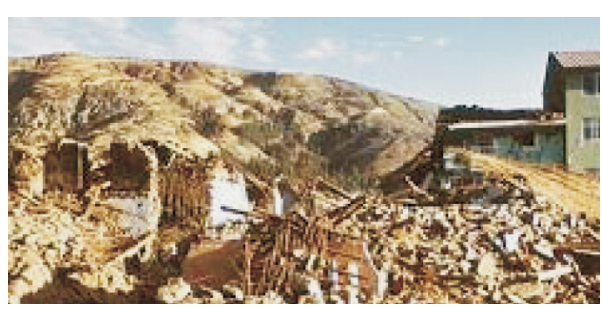

5. Casas de adobe destruidas por los sismos de Huaraz, 1970 (Fuente: NISEE) y de Pisco, 2007

6. Refuerzo de caña en muros de Caral, Perú

7. Nichos con caña de Chan Chan, Perú

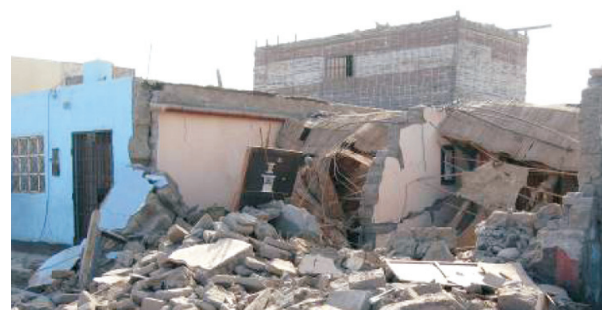

\section{TIERRA ARMADA}

Se denomina tierra armada al material compuesto de tierra y refuerzos de materiales compatibles y resistentes a tracción, que forman un conjunto desde el punto de vista mecánico y aprovechan con ventaja las propiedades resistentes de ambos materiales. La tierra armada es un material que trabaja esencialmente micro-fisurado y tiene un buen comportamiento sísmico gracias a la resistencia a la tracción y al control de deformaciones y desplazamientos que le confieren los refuerzos provistos.

Hasta la fecha, se han probado experimentalmente varios materiales compatibles con la tierra, como cañas, sogas naturales o artificiales, tubos de PVC y mallas plásticas (2). Se podría estudiar también la posibilidad de utilizar elementos delgados de madera, fibras vegetales, ramas de árboles u otros materiales sintéticos flexibles y resistentes como la fibra de vidrio.

Los refuerzos de las construcciones en tierra armada deben ser continuos en todos los muros porque las acciones sísmicas pueden causar esfuerzos de tracción en cualquier parte de la estructura. Los refuerzos podrán ser internos o externos, deben estar colocados en ambas caras de los muros y estar completamente recubiertos con un enlucido que los integre a los muros y los proteja de las acciones del medio ambiente.

Existen testimonios históricos de la durabilidad de los refuerzos de caña en Caral y Chan Chan (Figuras 6 y 7), donde se han encontrado vestigios de tecnologías semejantes a la tierra armada que han durado hasta la fecha. En la época colonial y republicana se usó también los tejidos de totora (un tipo de junco más blando y flexible que la caña) embutidos en las tortas de barro que cubrían los techos de las construcciones de la costa peruana.
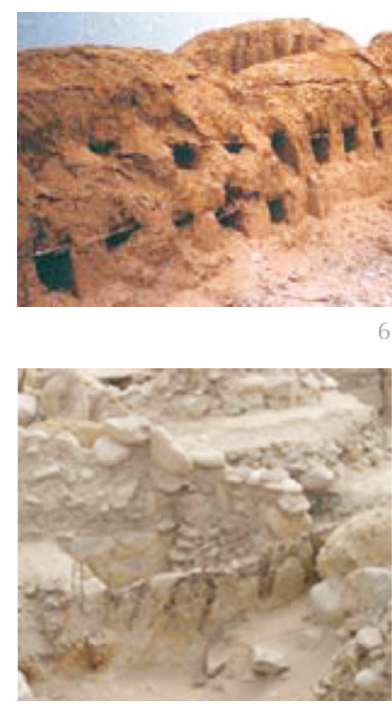


\section{Daños en viviendas de Pisco (Perú) \\ 9. Grietas verticales en las uniones de muros. \\ 5. VULNERABILIDAD DE LAS EDIFICACIONES DE TIERRA SIMPLE: EL TERREMOTO DE PISCO (PERÚ) DEL 2007}

10. Colapso de las fachadas en viviendas de adobe al voltearse hacia el exterior

11. Techo en casas de adobe 11(1) techo apoyado sobre fachada

11(2) techo no apoyado en fachada
El 15 de agosto del 2007 un sismo de magnitud Mw igual a 8,0 sacudió la zona centro sur del Perú. Se registraron casi 600 muertos, 50.000 viviendas colapsadas y más de 45.000 viviendas afectadas (3). La gran mayoría de daños se produjeron en las viviendas de adobe, tanto antiguas como nuevas (Figura 8) y en los monumentos históricos como iglesias construidas con tierra o con ladrillo. Las edificaciones de albañilería o de concreto armado sufrieron en general poco daño, salvo en casos en los que fueron mal diseñadas o construidas (3).

En la ciudad de Pisco, casi el $80 \%$ de las viviendas de adobe fue destruído, debido principalmente a la falta de refuerzo sísmico. Otros factores influyentes en el colapso de las construcciones fueron el suelo blando
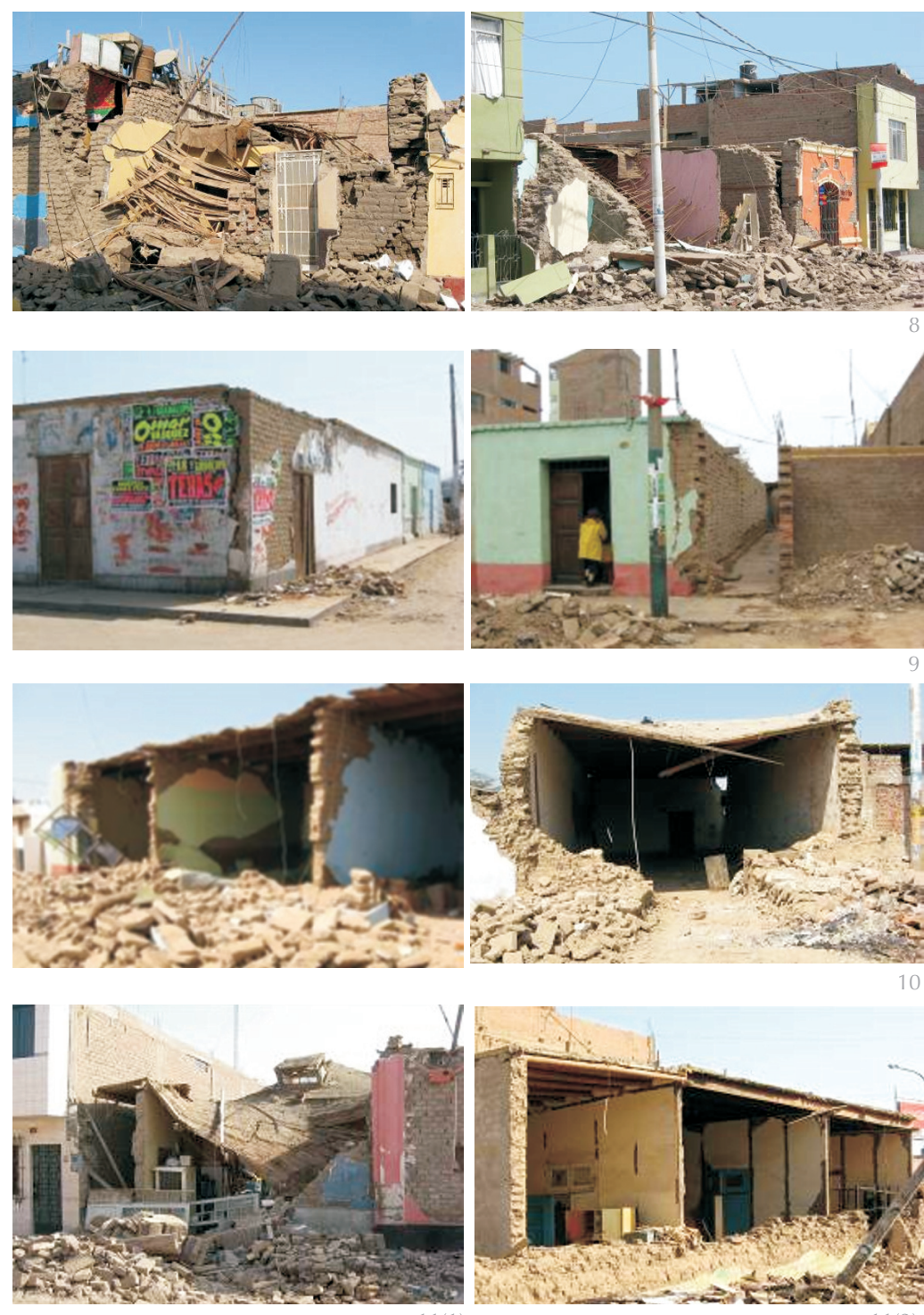

bajo la cimentación, la baja calidad de los materiales y mano de obra, el poco espesor de los muros, la inadecuada configuración de los vanos, y la débil conexión entre muros y entre muros y techos. El proceso de colapso sísmico se inicia usualmente con la aparición de grietas verticales en los encuentros de muros (Figura 9), que causa la desconexión de los muros, el desplome de la fachada debido al impacto de los muros perpendiculares (3), Figura 10, y por último la caída de los techos.

El tipo de daño producido por el colapso del muro de fachada depende de si las viguetas del techo están apoyadas o no en el muro de fachada. Si están apoyadas sobre la fachada, entonces al colapsar la fachada las viguetas pierden el equilibrio y se produce también la caída del techo (Figura 11a). En cambio, si las viguetas están apoyadas sobre los muros perpendiculares a la fachada, el techo por lo general no colapsa (Figura 11b).

Las viviendas ubicadas en esquina sufrieron daños debido al colapso de sus dos fachadas y del techo. Las fisuras que se produjeron en la zona de unión de las dos fachadas fueron verticales y diagonales. El patrón de falla diagonal que se forma va desde la parte superior de cada muro de esquina hacia la base de la casa formando una "V" (Figura 12).

Aquellos muros que no fallaron por volteo pudieron resistir cargas actuando en su propio plano y fallaron por corte. Se observaron algunos de estos casos en los que aparecieron grietas diagonales características (Figura 13). Las esquinas de los vanos, como puertas y ventanas, son los lugares donde se generan concentraciones de esfuerzos a tracción y es ahí donde se inician las grietas.

\section{INVESTIGACIONES DE LA PUCP EN TIERRA ARMADA}

\section{Refuerzo sísmico en casas de tierra}

Desde la década del 70 los profesores del Departamento de Ingeniería de la PUCP han tenido la preocupación de investigar la estabilidad de las construcciones de tierra en áreas sísmicas. Los primeros trabajos se orientaron a determinar las características mecánicas de la mampostería de adobe mediante ensayos estáticos de muros. Entre 1973 y 1978 se utilizó una plataforma inclinable (Figura 14) para ensayar estáticamente módulos de vivienda a escala natural y probar distintos materiales de refuerzo, como caña, madera y alambre (4). También se estudió el comportamiento mecánico de la mampostería de adobe mediante ensayos de compresión, de tracción (tipo brasilero), de compresión diagonal en muretes, y de corte y flexión en muros a escala natural (5). 
El refuerzo más eficiente fue logrado mediante la colocación de cañas verticales enteras al interior de los muros, espaciadas $1 \frac{1}{2}$ veces el espesor de los muros, y amarradas a franjas de caña chancada colocadas cada cuatro hiladas de mortero. Las mallas de refuerzo interior de caña natural mejoraron la resistencia y la capacidad de deformación de los muros y los módulos de adobe ensayados (Figura 15).

Como parte de un programa de investigación sobre Vivienda Económica Urbana (6), se inició un estudio del mortero de barro. Se construyeron y ensayaron al corte (Figura 16) catorce muros de tamaño natural. Por tratarse de un adobe urbano, se emplearon alternativas de mejora del mortero de barro común mediante la adición de materiales como cal, yeso y cemento. Los estabilizadores produjeron morteros más resistentes que un mortero de tierra pobre. Más tarde se descubrió que eran superados por morteros de tierra arcillosa con paja o arena gruesa, y por ello su costoso uso no es recomendable.

En 1983 se desarrolló un proyecto con el financiamiento de USAID para entender mejor la influencia de las propiedades de los materiales en la resistencia de la mampostería de adobe (7 y 8). Se realizaron 246 ensayos de muretes en compresión diagonal (Figura 17a) y cinco muros a escala natural (Figura 17b). Las principales conclusiones fueron que el mortero es responsable de la integración de la mampostería, que la arcilla es el componente más importante del suelo para construir con tierra ya que provee la ligazón entre mortero y adobes, que sin embargo la arcilla produce la contracción por secado que causa las fisuras del mortero, y que se puede controlar estas fisuras mediante la adición de paja o arena gruesa al mortero.

También se realizaron los primeros ensayos sísmicos en módulos de viviendas de adobe utilizando la mesa vibradora unidireccional de la PUCP (9). Se ensayaron módulos de vivienda sin techo, con y sin refuerzo interior de caña, en concordancia con las recomendaciones del Reglamento Nacional de Construcciones del Perú (10). Los módulos reforzados tuvieron varas verticales de caña colocadas cada 0,45 m y bandas de caña chancada horizontal cada cuatro hiladas, además de una viga solera superior de madera. Los módulos fueron instrumentados con sensores de desplazamiento y de aceleración y sometidos a sismos simulados de amplitud creciente. La principal conclusión fue que ante un sismo severo las construcciones no reforzadas colapsan luego de la separación de los muros en las esquinas (Figura 18a). El refuerzo interior de caña hori- zontal y vertical, combinado con una viga de coronación de madera, impide la separación de los muros y mantiene la integridad ante repetidos sismos severos unidireccionales (Figura 18b). Estos resultados ratificaron
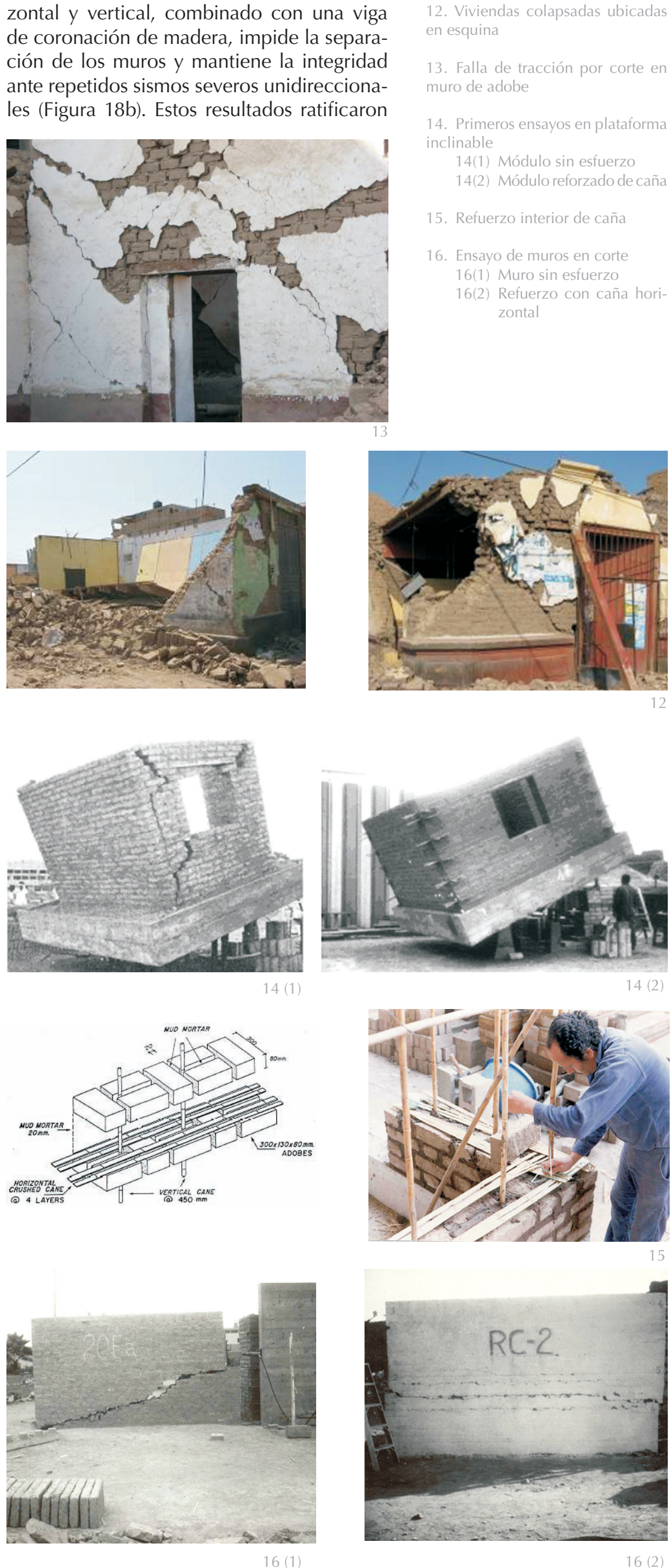


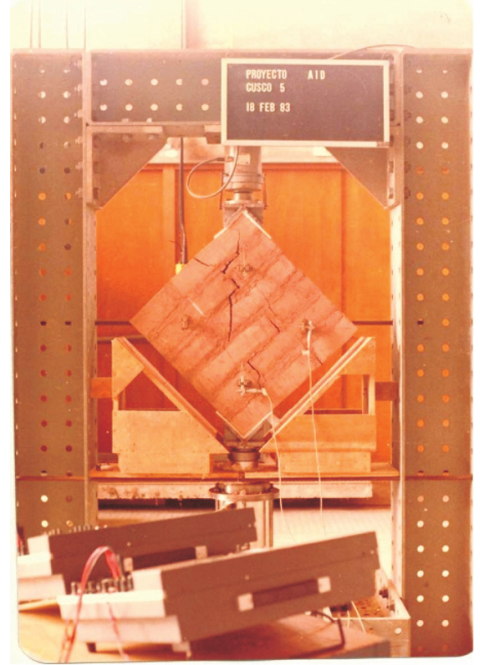

17(1)

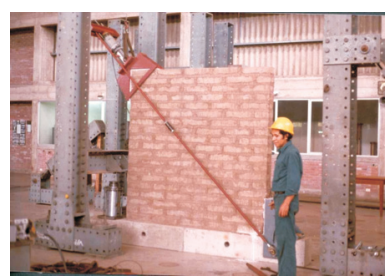

$17(2$

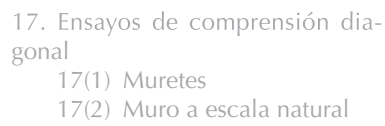

18. Ensayos en mesa vibradora de módulos de adobe

18(1) Colapso en módulo sin refuerzo

18(2) Daño en módulo reforzado

19. Ensayos de módulos de adobe reforzados con malla gallinero (izq.) y con soga (der.)

20. Ensayo de muro con refuerzo exterior de geomalla

21. Vivienda reforzada con malla electrosoldada después del sismo de junio del 2001, Perú (14)
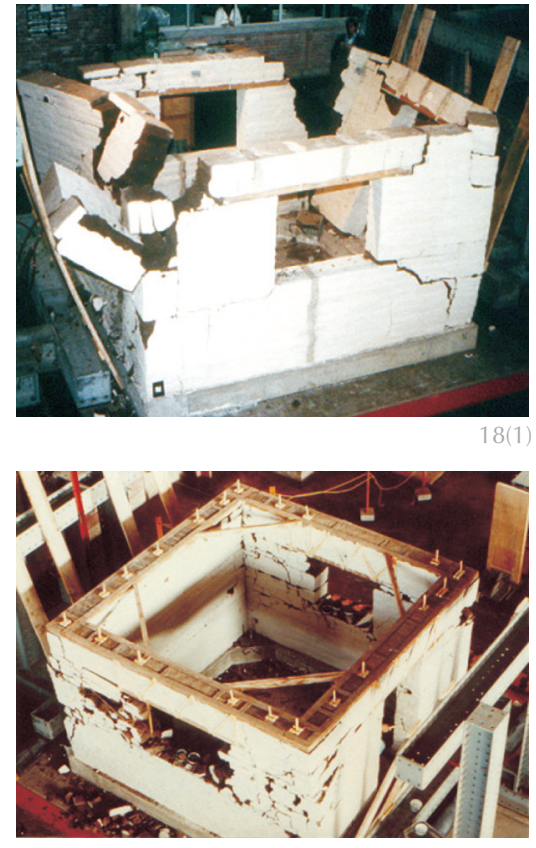

18(2)

la eficacia de la tierra armada con mallas internas de caña para evitar la falla frágil de las construcciones de tierra simple.

La caña, sin embargo, es difícil de conseguir en cantidad suficiente para la ejecución de programas masivos de construcción de viviendas y por ello es necesario contar con alternativas en otros materiales.

En 1994 se inició un proyecto financiado por la Cooperación Alemana al Desarrollo (GTZ) para estudiar posibilidades de refuerzo para las viviendas de tierra existentes. Se buscó aumentar la resistencia sísmica de las viviendas de tierra mediante refuerzos externos, que retardarían el colapso sísmico de
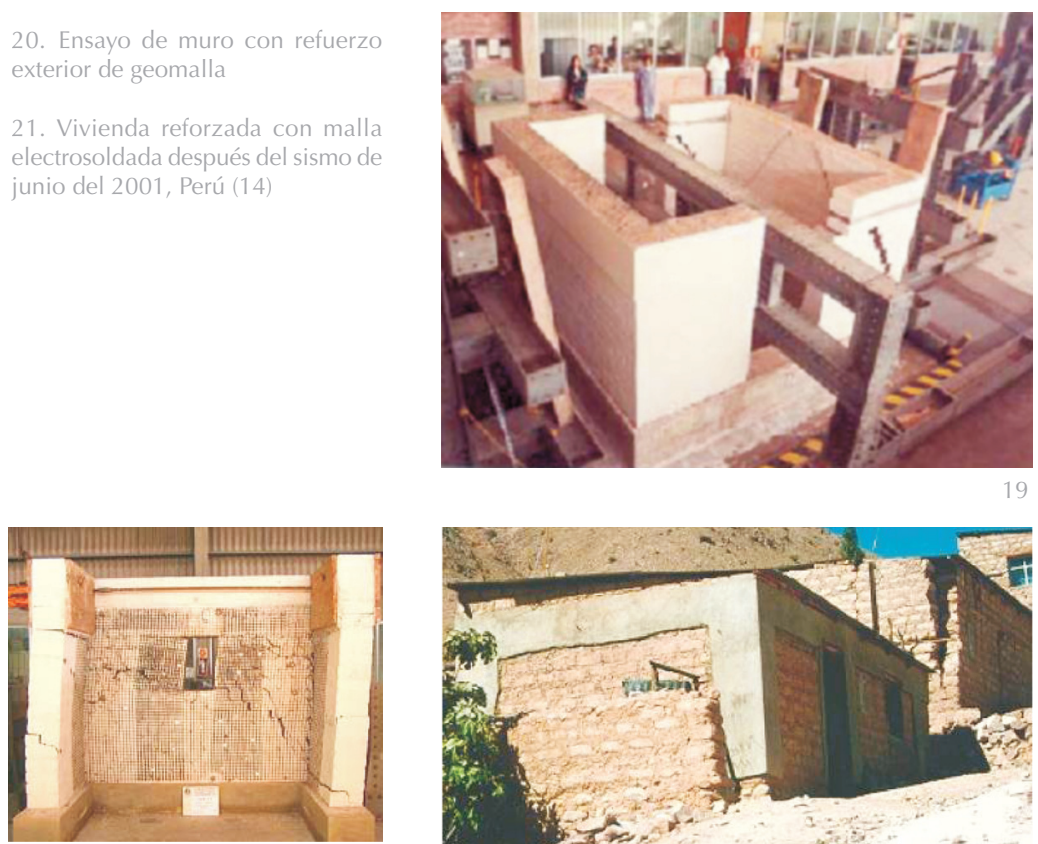

las viviendas para así salvar la vida de sus ocupantes. Entre 1994 y 1997 se hicieron ensayos de simulación sísmica en seis muros en forma de $U$ (Figura 19) y en cinco módulos de vivienda. Se probaron refuerzos exteriores utilizando sogas, tablas de madera, mallas de gallinero y mallas electrosoldadas de acero.

En este programa la solución más eficiente fue la de refuerzo exterior colocado en ambas caras de los muros, consistente en franjas de malla electrosoldada embutidas en un tarrajeo de cemento y arena y ubicadas verticalmente en las esquinas y horizontalmente en la parte superior de los muros. El sistema desarrollado aumenta el nivel de seguridad sísmica de las viviendas de tierra, ya que se incrementa la resistencia de los muros, se controla su desplazamiento y se pospone el colapso (11), (12), (13). Se logró, por tanto, el objetivo propuesto porque el sistema de refuerzo permitiría que las casas de tierra puedan resistir sismos leves y moderados. Sin embargo, es probable que no se llegue a evitar el colapso de las viviendas en terremotos severos, y además el modo de falla sigue siendo súbito y frágil. Este sistema se implementó en varios programas pilotos de reforzamiento de viviendas en el sur del país y en Bolivia y Chile. En el terremoto del 23 de junio del 2001 (Mw 7,9) las viviendas reforzadas en el programa piloto soportaron sin daños este movimiento sísmico moderado, mientras que construcciones cercanas de adobe tradicional colapsaron (Figura 21), (14).

Hace ya cerca de 5 años se inició una nueva línea de investigación para desarrollar sistemas de refuerzo de muros de adobe más eficientes utilizando materiales industrializados. Un primer proyecto encontró que los materiales industriales permiten lograr un comportamiento sísmico comparable al obtenido con los refuerzos de caña, con lo que se ha corroborado la posibilidad del uso de refuerzos plásticos (Figura 20) para conseguir construcciones sismorresistentes de adobe (2). Se encontró que los materiales industriales aumentan la resistencia y la capacidad de deformación de los muros y se consigue un comportamiento sísmico comparable al obtenido con los refuerzos de caña, y superior al obtenido con el refuerzo de malla electrosoldada.

Con el apoyo del Instituto Getty de Conservación $(\mathrm{GCl})$ se realizaron ensayos de simulación sísmica en dos módulos reforzados: uno con malla de plástico colocada en toda la superficie exterior e interior de los muros y el otro con refuerzo exterior de caña vertical y soga horizontal. En ambos casos se colocaron estucos sólo a medio módulo 
(Figura 22a). Este proyecto ha demostrado la importancia de tener los refuerzos embutidos en estucos de barro para lograr el trabajo conjunto de tierra seca y refuerzo, con lo que se consigue un excelente desempeño sísmico de los muros (15). Los refuerzos estudiados colocados sin recubrimiento mejoran la capacidad de deformación, pero no la resistencia cortante y de flexión de los muros. En cambio, cuando estos mismos refuerzos están embebidos dentro de los estucos de barro, se consigue una mejora notable, tanto en la capacidad de deformación como en la resistencia de los muros. Los estucos son, por tanto, indispensables para conseguir un buen control de la fisuración y para evitar el colapso de los muros. Se observó además que en los muros sin estuco, las grietas producidas en los muros tuvieron un espesor mayor a las producidas en los muros revestidos.

Posteriormente se han hecho más ensayos de simulación sísmica con ayuda de la Dirección Académica de Investigación (DAI) de la PUCP. Estos ensayos tuvieron como finalidad optimizar la cantidad de geomalla colocada en los muros (Figura 22b) y evaluar el uso de mallas plástica económicas, como la usada como malla de seguridad en sitios en construcción (Figura 23), (16).

Cantidades moderadas de malla colocadas estratégicamente en los muros podrían ser usadas para evitar el colapso parcial o total de las viviendas de tierra. La malla debe ser colocada en ambas caras de los muros, conectadas incluso a través de los muros y recubiertas con estuco. La disminución de refuerzo, sin embargo, controla menos eficientemente los desplazamientos; es decir, significa aceptar un mayor nivel de daños que podrían convertirse en irreparables. De los últimos ensayos se concluye que las mallas económicas no estandarizadas no son confiables y además, que no es recomendable colocar mallas de refuerzo que dejen áreas de muros sin refuerzo.

\section{Reparaciones en muros de tierra}

A partir del 2006 se inició una investigación para lograr eficientes reparaciones de fisuras en muros de tierra a través de métodos de inyección de lechadas (grouts) de barro, con materiales que conlleven la menor alteración posible al material patrimonial de tierra. Esta investigación se realizó con el apoyo de la DAI de la PUCP. Como resultado del primer año de investigaciones, se comprobó que utilizando barro líquido conformado por suelos tamizados por la malla \# 10 (ASTM E11-04) y un contenido de agua del $30 \%$, era posible reparar fisuras y restituir la resistencia original en los muretes de mampostería de tierra.
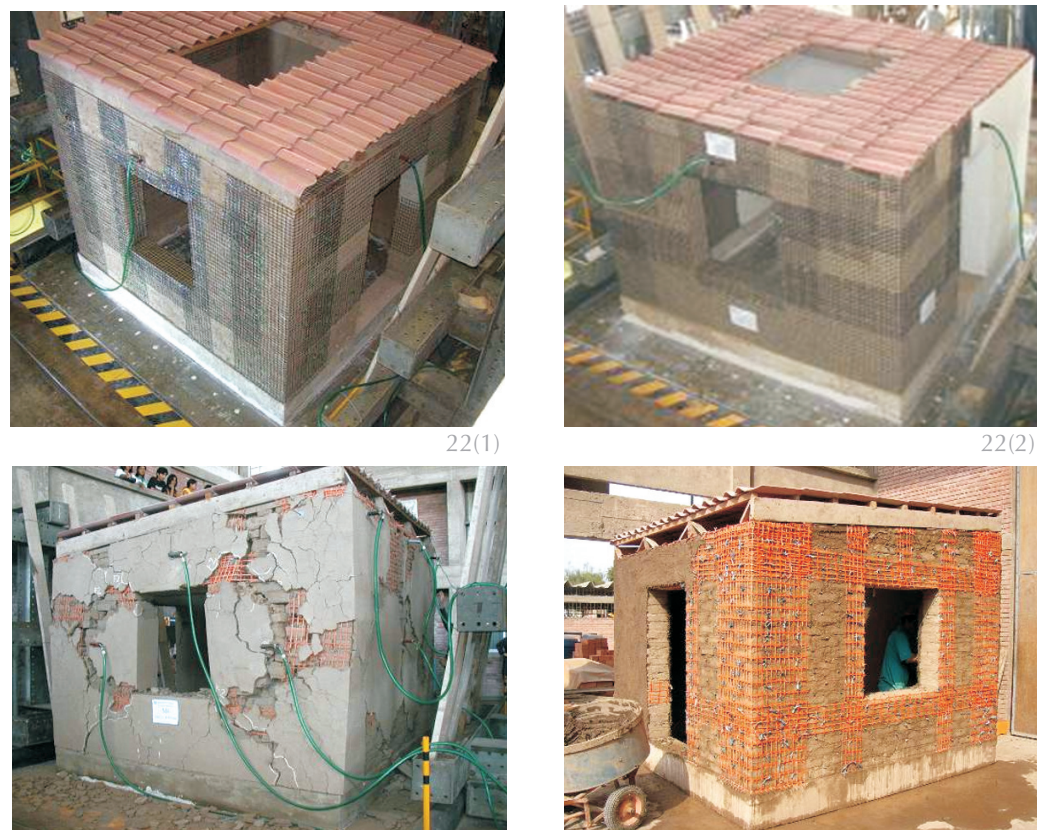

Se fabricaron muretes de albañilería de adobe que se ensayaron a compresión diagonal hasta producir la falla con agrietamiento (Figura 24). Luego, se repararon por inyección las fisuras (Figura 25), se dejó secar y se volvió a ensayar el espécimen reparado (Figura 26). Se puede apreciar que la fisura de la falla sigue una trayectoria diferente, que corresponde a una carga superior a la original (17) .

El problema de mayor dificultad en la inyección de fisuras es la penetrabilidad del barro líquido. Mientras más finas sean las grietas, más fino y fluido debería ser el grout .Era necesario conocer las leyes del agrietamiento del grout (en elementos muy delgados) durante su secado dentro de una fisura de un muro, comparándolo con el comportamiento de filmes de barro, que se secaran en condiciones controladas pero variables. Por ello se decidió investigar el secado y agrietamiento de filmes de barro de tierra tamizada y agua.
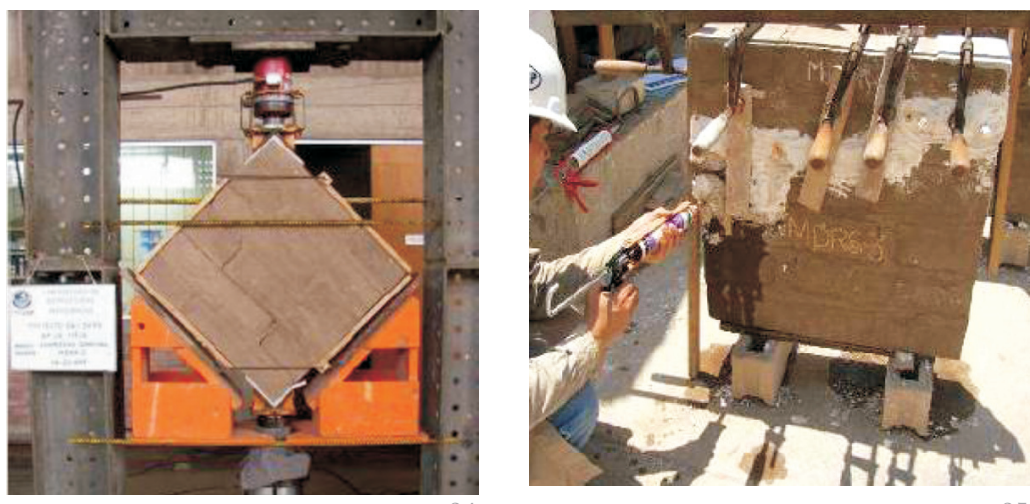


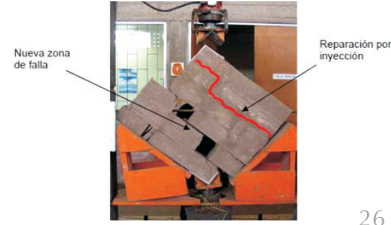
del murete reparado

27. Casa Marrou

28. Huaca Mateo Salado

29. Ilustraciones del manual de construcción

30. Clases prácticas de construcción en el campus de la PUCP
26. Rotura por diferente trayectoria

En el año 2007, con el apoyo del Instituto Getty de Conservación $(\mathrm{GCl})$, se realizaron trabajos de laboratorio consistentes en el estudio por observación de filmes de barro líquido depositado entre dos papeles celofán y dos piezas de vidrio, que permitieron comprender relaciones entre la formación de fisuras y la velocidad de secado, influencia del espesor de los filmes, importancia del contenido de agua y de la finura del tamizado. Se encontró que la velocidad de secado es una variable crucial en la formación de fisuras. A menor velocidad de secado, menos número de fisuras o menor espesor de fisuras, es decir, menor agrietamiento. Los filmes delgados tienen menos imperfecciones y mayor uniformidad, lo que los convierte en más resistentes (17).

En el 2008, miembros del equipo de investigación empiezan a realizar las primeras aplicaciones y difusión del sistema de reparación por inyección de barro tamizado líquido en sitios históricos situadas en Lima como casonas del centro de la ciudad o del antiguo barrio de Barranco, la huaca de Mateo Salado y el Acllawasi de Pachacamac, a través de colaboraciones de asesoría o de consultorías privadas, realizadas al amparo de un convenio entre la PUCP y el Instituto Nacional de Cultura.

En la Figura 27 se aprecia la puesta en valor de la casa Marrou en Barranco, donde se re-
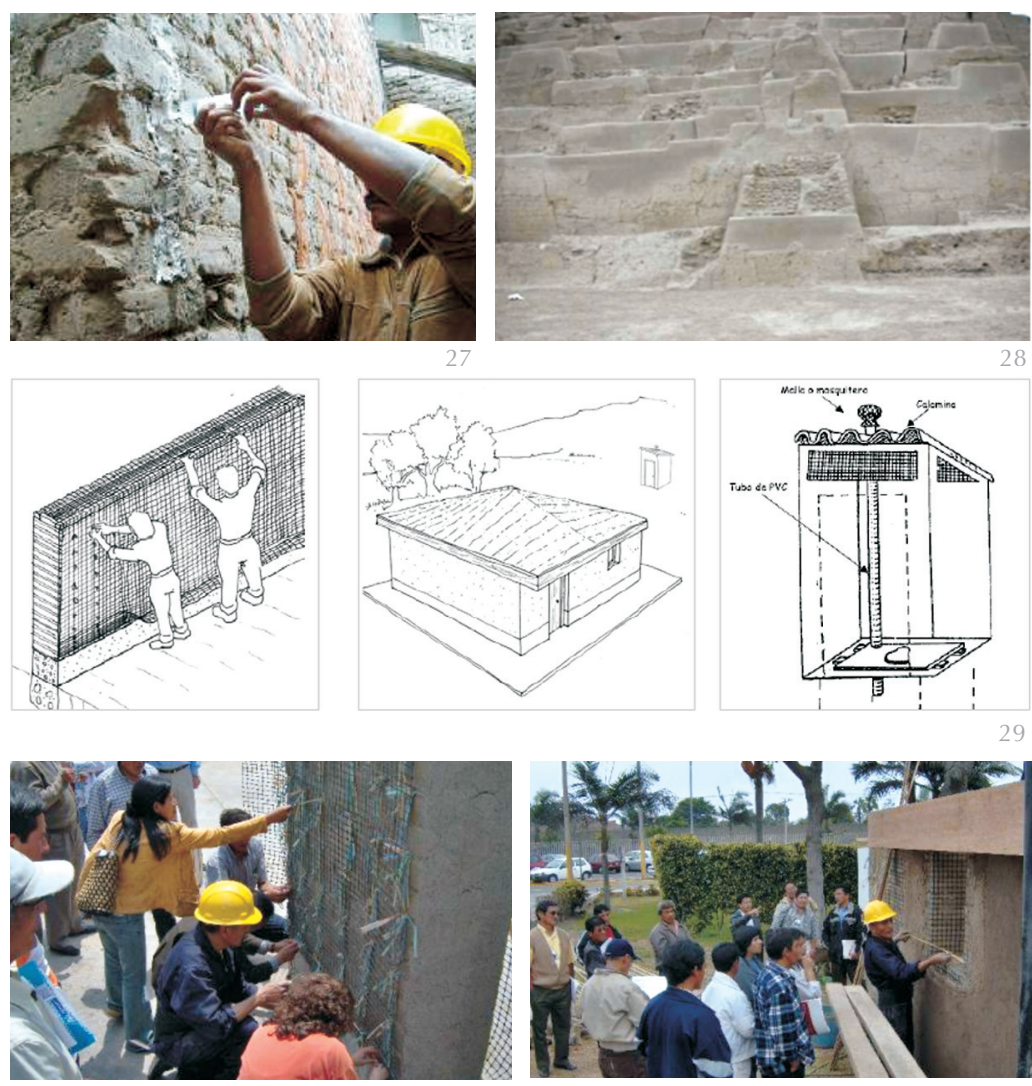

comendó la reparación de fisuras por inyección de barro líquido. En la Figura 28 se ve una de las fachadas de la Huaca Mateo Salado, cuya consolidación requirió inyección de lechadas de barro para recuperar la estabilidad de los muros de sostenimiento perimetrales en los distintos niveles. También en otros sectores se rehizo, por métodos de anastilosis e inyecciones de barro líquido, algunos muros utilizando las partes originales agrietadas (18).

Las mayores dimensiones de las obras en el campo llevaron también a tecnificar los procedimientos de inyección, pues el caso de inyectar muros de tierra es un caso único entre el grupo de las albañilerías de materiales más fuertes (ladrillo, piedra con morteros de cal o cemento). La experiencia ganada en inyecciones de otros materiales internacionalmente, no es directamente aplicable y la realizada en tierra no siempre garantiza la restitución de su capacidad resistente original. La práctica de campo ha permitido perfeccionar los procedimientos planteados desde el laboratorio. Actualmente se intenta mejorar los procedimientos de inyección de barro líquido, en ensayos cíclicos y dinámicos en modelos a escala natural. A la fecha, ya se han realizado ensayos de módulos de 4 paredes en la mesa vibradora de la PUCP, agrietando los muros en una primera fase de ensayos, reparándolos después y finalmente volviéndolos a ensayar para comparar los resultados y restitución de resistencia.

\section{EXPERIENCIA SOBRE RECONSTRUCCIÓN}

Después del terremoto de Pisco la PUCP, basada en las investigaciones previas, y conjuntamente con la organización no gubernamental de ayuda para el desarrollo CARE Peru propusieron un modelo de casa de adobe reforzado con geomalla. La propuesta también incluyó el desarrollo de material educativo, estrategias de diseminación sobre la construcción de casas sismorresistentes, la capacitación sobre construcción a los pobladores, logística de materiales y supervisión de la construcción (19).

Como propuesta de diseminación de la información, la PUCP y CARE Perú, conjuntamente con el apoyo del SENCICO y FORSUR (organizaciones gubernamentales), desarrollaron dos manuales de construcción saludable y sismorresistente de viviendas de adobe reforzadas con geomalla. Uno de los manuales fue para la construcción con adobe en regiones áridas como la costa del Perú y el otro para regiones lluviosas, como la sierra del Perú. Los manuales explican de forma sencilla y con muchas ilustraciones la correcta forma de construcción de casas de 


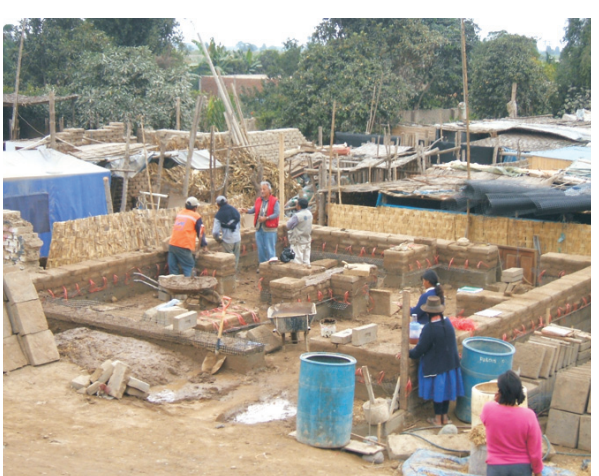

adobe (Figura 29). Los dos manuales son de libre acceso y disponibles en:

http://www.pucp.edu.pe/documento/publicaciones/construccion-de-casas-saludablesy-sismoresistentes-de-adobe-reforzado-zona-de-la-sierra.pdf

La estrategia de capacitación se realizó bajo el enfoque de aprendizaje en cascada. Al inicio se capacitó a pocas personas, quienes sirvieron como guiadores para la capacitación de más personas. Durante la primera fase del programa alrededor de 100 personas (constructores, albañiles, técnicos en construcción, ingenieros civiles, personal de organismos gubernamentales y no gubernamentales) fueron capacitadas en el campus PUCP. El proceso incluyó clases teóricas y prácticas con la construcción de una casa sismorresistente (Figura 30).

La segunda fase, con alrededor de 360 participantes, fue desarrollada en las ciudades más afectadas por el sismo (Figura 31). Materiales audiovisuales de la primera parte fueron usados para explicar el proceso de construcción. Los participantes construyeron nueve bajo la supervisión de la PUCP y CARE Perú, bajo fondos del FORSUR. Hasta la fecha se han culminado 2.000 casas de adobe sismorresistente. En abril del 2009, el Gobierno Peruano dio un decreto para crear un programa nacional de reconstrucción de viviendas de adobe sismorresistentes, seguras y saludables a un costo aproximado de \$ 600 por familia.

\section{COMENTARIOS FINALES Y RECOMENDACIONES}

Los trabajos realizados en los últimos 40 años en la PUCP, descritos en este artículo, han establecido fundamentos sólidos para el futuro desarrollo del material tierra armada con el que se pueden construir muros de

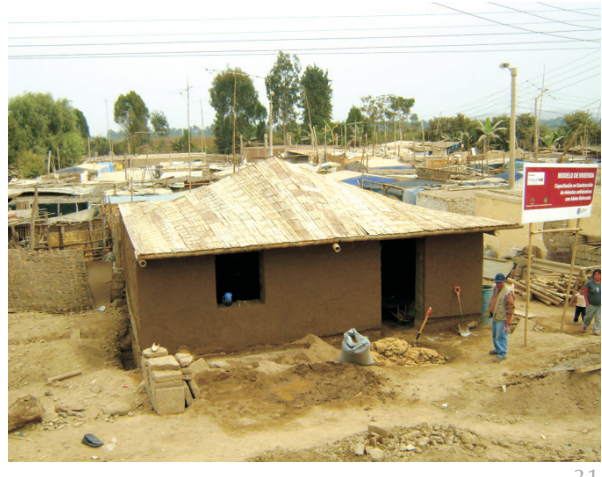

tierra sismorresistentes capaces de salvaguardar la vida de los habitantes. El conjunto tierra armada contiene elementos de refuerzo como cañas embutidas horizontal y verticalmente en lo muros, mallas electrosoldadas colocadas al exterior de los muros y recubiertas con mortero de cemento o maIlas plásticas colocadas también al exterior de los muros pero recubiertas con mortero de barro.

Durante un sismo, la fase de comportamiento elástico de los muros estructurales de tierra armada es muy breve, porque la tierra se fisura a un nivel muy bajo de esfuerzos de tracción. Se ha ya demostrado que la integración entre la tierra y los refuerzos permite un comportamiento adecuado de los muros en su fase inelástica.

La geometría de las nuevas edificaciones en tierra armada debe realizarse considerando muros anchos y poco esbeltos, plantas con habitaciones de preferencia cuadradas, distribución simétrica, y ventanas centradas y pequeñas, tal como se especifica actualmente para la construcción con tierra simple (20). Sin embargo, queda como tarea terminar de definir las especificaciones técnicas para el adecuado diseño sismorresistente en tierra armada.

En los trabajos de conservación de obras patrimoniales, es posible y conveniente utilizar también refuerzos compatibles, siempre y cuando se respeten los principios básicos de intervención contenidos en las cartas de conservación adoptadas por ICOMOSUNESCO, tales como la mínima intervención y reversibilidad de los refuerzos compatibles.

Nota- Todas la figuras en este artículo son de propiedad de los autores, excepto en las que se mencionan las fuentes.
31. Pobladores construyendo sus propias casas en Chincha, Ica

\section{Agradecimientos}

Este artículo resume los resultados de varios proyectos de investigación desarrollados en la Pontificia Universidad Católica del Perú, muchos de ellos con el apoyo económico de la Dirección Académica de Investigación (DAI). La continua contribución de Daniel Torrealva, Francisco Ginocchio y Gladys Villa-García, profesores de la PUCP, es invaluable. El Instituto Getty de Conservación, a través de Mary Hardy y Claudia Cancino, también ha sido partícipe de los últimos proyectos de investigación. Por último, los autores expresan su gratitud al personal del Laboratorio de Estructuras de la PUCP y a los estudiantes que han trabajado arduamente durante el desarrollo de los proyectos: Ivonne Madueño, José Acero, José Velásquez, María Ángela Astorga, Katia Morales, Julio César Chang, Carlos Fernández, Karina Sánchez, Álvaro Rubiños, Denisse Palomino y Lizet Vargas. También se agradece a la Dirección Académica de Responsabilidad Social (DARS), por la organización social de la etapa de capacitación en el campo y difusión de la tecnología de geomallas polímeras. 


\section{BIBLIOGRAFÍA}

(1) INEI: "Censos Nacionales 2007: XI de Población y VI de Vivienda". Instituto Nacional de Estadística e Informática, http://iinei.inei.gob.pe/iinei/RedatamCpv2007.asp?ori=C. 2008, Lima, Perú.

(2) Blondet, M.; Torrealva, D.; Villa-García, G.; Ginocchio, F. y Madueño, I.: "Uso de materiales industriales para la construcción de viviendas seguras de adobe" (en inglés). Memorias del Congreso Internacional EarthBuild 2005, pp. 76-90. NSW: Faculty of Design, Architecture and Building, University of Technology, Sydney, 2005.

(3) Blondet, M.; Vargas, J. y Tarque, N.: "Comportamiento sísmico de las casa de tierra durante el terremoto de Pisco" (en inglés). Memorias del 14 Congreso Mundial en Ingeniería Sismorresistente. International Association of Earthquake Engineering IAEE, Chinese Association of Earthquake Engineering CAEE, paper ID S29-008, Beijing, 2008.

(4) Corazao, M.; Blondet, M.: Estudio experimental del comportamiento estructural de las construcciones de adobe frente a solicitaciones sísmicas. Banco Peruano de los Constructores, Lima, 1973.

(5) Blondet, M.; Vargas, J.: "Investigación sobre vivienda rural". Convenio con el Ministerio de Vivienda y Construcción. Departamento de Ingeniería, Sección Ingeniería Civil, Publicación DI-78-01, PUCP, Lima, 1978

(6) Vargas, J.: "Albañilería de adobe con variaciones de mortero". Departamento de Ingeniería, Sección Ingeniería Civil, Publicación DI-79-02, PUCP, Lima, 1979.

(7) Vargas, J.; Bariola, J.; Blondet, M.; Villa-García, G.; Ginocchio, F.: "Propiedades del suelo para elaborar albañilería de adobe". Memorias del Seminario Latinoamericano de Construcciones Sismo-Resistentes de Tierra. Departamento de Ingeniería, Sección Ingeniería Civil, PUCP, 585-626, Lima, 1983.

(8) Vargas, J.; Bariola, J.; Blondet, M.; Villa-García, G.; Ginocchio, F.: "Investigación científica innovativa: Edificaciones de adobe en áreas sísmicas". Departamento de Ingeniería, Sección Ingeniería Civil, Proyecto AID 936/5542, PUCP, Lima, 1983.

(9) Vargas, J.; Bariola, J.; Blondet, M.: "Resistencia sísmica de la mampostería de adobe". Departamento de Ingeniería, Sección Ingeniería Civil, Publicación DI-84-01, PUCP, Lima, 1984.

(10) OIN. RNC: Normas de diseño sismo-resistente. Oficina de Investigación y Normalización. Ministerio de Vivienda y Construcción, Lima, 1977.

(11) Zegarra, L.; Quiun, D.; San Bartolomé, A.; Giesecke, A.: "Reforzamiento de viviendas existentes de adobe". Proyecto Centro Regional de Sismología para América del Sur (CERESIS) - Cooperación Alemana al Desarrollo (GTZ) - Pontificia Universidad Católica del Perú (PUCP). Memorias del XII Congreso Nacional de Ingeniería Civil, pp. 187-192. Colegio de Ingenieros del Perú, Huanuco, 1999.

(12) Zegarra, L.; Quiun, D.; San Bartolomé, A.; Giesecke, A.: "Reforzamiento de viviendas de adobe existentes. Primera parte: Ensayos sísmicos de muros U". Memorias del XI Congreso Nacional de Ingeniería Civil, pp. 172-196. Colegio de Ingenieros del Perú, Trujillo, 1997.

(13) Zegarra, L.; Quiun, D.; San Bartolomé, A.; Giesecke, A.: "Reforzamiento de viviendas de adobe existentes. Segunda parte: Ensayos sísmicos de módulos". Memorias del XI Congreso Nacional de Ingeniería Civil, pp. 177-181. Colegio de Ingenieros del Perú, Trujillo, 1997

(14) Zegarra, L.; Quiun, D.; San Bartolomé, A.: "Comportamiento ante el terremoto del 23-06-2001 de las viviendas de adobe reforzadas en Moquegua, Tacna y Arica". Memorias del XIII Congreso Nacional de Ingeniería Civil, pp. 16-18. Colegio de Ingenieros del Perú, Puno, 2001.

(15) Torrealva, D.; Acero, J.: "Refuerzo sísmico de vivienda de adobe con malla exterior compatible". Memorias del Seminario Internacional de Arquitectura, Construcción y Conservación de Edificaciones en Tierra en Áreas Sísmicas, SismoAdobe2005, PUCP, http://www.pucp.edu.pe/eventos/SismoAdobe2005/. 2005, Lima, Perú.

(16) Blondet, M.; Vargas, J; Tarque, N.; Velásquez, J.: "Estudio experimental de mallas sintéticas como refuerzo sísmico en muros de adobe" (en inglés). Memorias del SAHC2006, pp. 709-716. Macmillan India Ltd. Vol 2, New Delhi, 2006.

(17) Blondet, M.; Vargas, J.; Ginocchio, F.; Morales, K.; Iwaki, C.: "Estudio preliminar del uso de grouts de barro para reparar fisuras estructurales en muros históricos de adobe" (en inglés). Memorias de AdobeUSA2007, pp. 52-59. The Adobe Association of the Southwest, El Rito, New Mexico, 2007.

(18) Blondet, M.; Vargas, J.; Tarque, N.: "Tecnología de bajo costo para mejorar el desempeño sísmico de viviendas de adobe en países en desarrollo" (en inglés). Memorias del 14 Congreso Mundial en Ingeniería Sismorresistente. International Association of Earthquake Engineering IAEE, Chinese Association of Earthquake Engineering CAEE, paper ID 09-02-001, Beijing, 2008.

(19) Blondet, M.; Vargas, J.; Patron, P.; Stanojevich, M.; Rubiños, A.: "Desarrollo humano para la construcción de viviendas seguras e higiénicas en áreas sísmicas" (en inglés). Memorias del Congreso Internacional Kerpic08-Learning from earthen architecture in climate change. Universidad Internacional de Chipre. paper ID 45, Lefkosa, 2008.

(20) MTC. Reglamento Nacional de Construcciones. Adobe: Norma Técnica de Edificación E-080. Ministerio de Transportes, Comunicación, Vivienda y Construcción (MTC). Servicio Nacional de Capacitación para la Industria de la Construcción (SENCICO), Lima, 2000.

$$
* * *
$$

